\title{
Ansiedad y calidad de vida en mujeres con cáncer de mama: una revisión teórica
}

\section{Anxiety and quality of life in women with breast cancer: A theoretical review}

\author{
Cecilia Mota González ${ }^{1}$, Evangelina Aldana Calva ${ }^{1}$ \\ Lyda M. Bohórquez Zambrano ${ }^{2}$, Sandra Martínez Medina ${ }^{3}$ \\ y José Raúl Peralta López ${ }^{4}$
}

\begin{abstract}
RESUMEN
El cáncer de mama es actualmente un padecimiento que afecta a un gran número de personas en el mundo, ocurriendo en el sexo femenino la mayor incidencia. En países como México, se reconoce el cáncer de mama como la segunda causa de muerte en las mujeres adultas. Este artículo hace una breve revisión de la literatura sobre esta condición médica, sus implicaciones psicológicas, el desarrollo de la consecuente ansiedad y su impacto en la calidad de vida. Se empleó la base de datos SCOPUS como principal herramienta, en la que se revisaron los artículos publicados entre 2012-2016, siendo los criterios de búsqueda los conceptos "cáncer de mama", "ansiedad" y "calidad de vida". Los estudios analizados evidencian que las personas con cáncer suelen generar el trastorno de ansiedad durante el diagnóstico, el tratamiento o la cirugía, lo que provoca un cambio considerable en su calidad de vida. La sintomatología propia de los trastornos de ansiedad suele acentuarse, por lo que se requiere la intervención de un especialista para disminuir el malestar derivado. La importancia de la intervención psicológica se evidencia al lograr la disminución de los síntomas, favoreciendo así el proceso de afrontamiento de la enfermedad y contribuyendo a una mejor calidad de vida de las pacientes.
\end{abstract}

Palabras clave: Ansiedad; Calidad de vida; Cáncer de mama.

\begin{abstract}
Currently breast cancer is a condition that affects a large number of people in the world, and women show the highest incidence. In countries like Mexico, breast cancer is recognized as the second cause of death in adult women. This article informs of a brief review of the research literature on this medical condition, its psychological implications, such as anxiety as a frequent component, and its impact on quality of life. The SCOPUS database was used as the main source, including articles published between 2012-2016. Search criteria included the concepts "breast cancer", "anxiety", and "quality of life". The studies analyzed show that people with cancer often develop anxiety disorders at the time of diagnosis. Treatment and surgery also involve conditions causing considerable deterioration in quality of life. All along these moments anxiety symptoms usually worsen and require the intervention of a specialist in order to reduce suffering and discomfort. The importance of psychological interventions is evidenced because they effectively reduce the symptoms, thus favoring the process of coping of the disease and contributing to improve patients' quality of life.
\end{abstract}

Key words: Anxiety; Quality of life; Breast cancer.

\footnotetext{
${ }^{1}$ Instituto Nacional de Perinatología, Coordinación de Psicología y Calidad, Montes Urales 800, Col. Lomas Virreyes, Del. Miguel Hidalgo, 11000 Ciudad de México, México, tel. (55)55-20-99-00, ext. 147, correo electrónico: motaceci@hotmail.com. Artículo recibido el 21 de junio y aceptado el 3 de octubre de 2017.

${ }^{2}$ Universidad Cooperativa de Colombia, Calle 20, Núm. 3027, Torre 1-1604, 680002 Bucaramanga, Colombia, tel. 57(315)801-64-92, correo electrónico: lydabohorquez@psicologos.com.

${ }^{3}$ Instituto Nacional de Perinatología, Departamento de Neurobiología del Desarrollo, Montes Urales 800, Col. Lomas Virreyes, Del. Miguel Hidalgo, 11000 Ciudad de México, México, tel. (55)55-20-99-00, ext. 470 correo electrónico: sandymarm@yahoo.com.mx.

${ }^{4}$ Universidad de Baja California, Calle Av. Juan F. Parkinson 160, Fraccionamiento Parque Ecológico, 63173 Tepic, Nay., México, tel. (311)133-42-42, correo electrónico: ubcposgrados@hotmail.com.

Citación: Mota G., C., Aldana C., E., Bohórquez Z., L.M., Martínez M., S. y Peralta L., J.R. (2018). Ansiedad y calidad de vida en mujeres con cáncer de mama: una revisión teórica. Psicología y Salud, 28(2), 155-165.
} 


\section{INTRODUCCIÓN}

$\mathrm{D}$ esde hace varias décadas el cáncer es considerado un importante problema de salud pública en todo el mundo, lo que plantea retos importantes en cuanto al diagnóstico y el tratamiento. Se ha encontrado que el aumento del cáncer en mujeres en los países de América Latina se asocia a patrones reproductivos, tales como edad de la menarca, edad del primer embarazo, número de partos y duración de la lactancia; en los países desarrollados está vinculado además con factores como el desarrollo económico, la urbanización, la obesidad, el consumo de alimentos procesados y el sedentarismo (González, García, Aguilar, Padilla y Álvarez, 2014; Lukong, 2017). Este padecimiento, al igual que todas las enfermedades crónicas, especialmente aquellas socialmente estigmatizadas, requiere una atención particular ya que moviliza diversas emociones, en especial en la dimensión subjetiva de la calidad de vida. El sufrimiento emocional de las mujeres que lo padecen se asocia a aspectos relacionadas con la cirugía, así como al significado que ellas atribuyen a estos sucesos (Martínez, Lozano, Rodríguez, Galindo y Alvarado, 2014).

El diagnóstico de cáncer de mama se considera un suceso estresante que no produce el mismo impacto en todos los individuos; sin embargo, si se le percibe como una amenaza, entonces ocurrirá una reacción emocional de ansiedad, que será más intensa en la medida en que el individuo perciba que sus recursos para afrontarla no son suficientes para controlar o manejar las consecuencias negativas que se prevén. Cuando la frecuencia, la intensidad o la duración de la respuesta de ansiedad es excesiva, tenderá a disminuir la capacidad de adaptación del individuo al medio circundante, $\mathrm{y}$ en consecuencia su bienestar psicológico se verá perturbado, y es entonces que se habla de una ansiedad patológica que afecta su vida personal, familiar, laboral o social.

El objetivo del presente trabajo es hacer una revisión teórica del papel que desempeña la ansiedad en la calidad de vida de mujeres con cáncer de mama, para lo cual se consultó como fuente y principal herramienta la base de datos SCOPUS, en la que se revisaron los artículos publicados entre los años 2012 y 2016, utilizando como criterios de búsqueda los conceptos "cáncer de mama", "ansiedad" y "calidad de vida". En dicha base se seleccionaron cuarenta artículos. De acuerdo a la revisión efectuada, se decidió presentar la información comenzando con la descripción y características del padecimiento, para después exponer las repercusiones emocionales, principalmente las relacionadas con la ansiedad y la calidad de vida, y finalmente hacer mención a los tipos de intervención psicológica que se pueden utilizar para disminuir la ansiedad en la mencionada condición.

\section{Etiología del cáncer de mama}

El cáncer de mama es una enfermedad crónico-degenerativa en la que se desarrollan células malignas en los tejidos de la mama, formando así tumores que invaden los tejidos vecinos y que pueden alcanzar órganos distantes. Es el tipo de tumor maligno más frecuente entre las mujeres en el mundo, representando $16 \%$ de todos los tipos de cáncer, y uno de los problemas de salud más importantes por sus efectos y frecuencia, la cual es más elevada entre las mujeres de 45 a 60 años de edad (Kern y Moro, 2012).

La incidencia del cáncer de mama varía considerablemente en todo el orbe, siendo mayor en los países desarrollados. En Europa, por ejemplo, se diagnostican aproximadamente 450 mil nuevos casos y la mortalidad se sitúa en torno a los 139 mil (Vich et al., 2014). El cáncer de mama se ha convertido en la primera causa de muerte debido a neoplasias en mujeres mexicanas desde el año 2006 (Torres et al., 2013).

Hasta la fecha todavía no se conocen exactamente las causas por las que se origina este padecimiento, pero se sabe que es un problema multifactorial y que el estrógeno desempeña un importante papel en su aparición ya que induce el crecimiento de las células mamarias (Martínez et al., 2014). Otros factores de tipo biológico relacionados con este tipo de cáncer son, a saber: mujeres con una edad mayor a 40 años, con antecedentes familiares de cáncer de mama, con cáncer de mama previo, sin hijos o que tuvieron a su primer hijo después de los 30 años de edad, con menarca ocurrida antes de los 12 años, con menopausia tardía después 
de los 52 años, así como con enfermedades benignas de la mama, como quistes (Vich et al., 2014).

Por otro lado, existen también factores de riesgo relacionados con el estilo de vida, tales como una dieta elevada en grasas, ingesta de alcohol, obesidad, tabaquismo y uso de hormonas por más de diez años (Vich et al., 2014).

\section{Etapas del cáncer de mama}

El cáncer de mama se clasifica por etapas usando el sistema TNM de la American Joint Commission on Cancer. Dicha clasificación está basada en el tamaño del tumor (T), la afectación de los ganglios linfáticos (N) y la metástasis en otras áreas del cuerpo (M). Además, según su propagación, se divide en los estadios que se muestran en el Cuadro 1.

Cuadro 1. Etapas del cáncer mamario.

\begin{tabular}{|c|l|}
\hline ESTADIO & \multicolumn{1}{c|}{ DESCRIPCIÓN } \\
\hline 0 & Las células malignas se mantienen dentro de la mama sin invadir el tejido normal próximo. \\
\hline I & El tumor mide $2 \mathrm{~cm}$ o menos, y entre uno y tres ganglios linfáticos axilares están invadidos por células anormales. \\
\hline II & $\begin{array}{l}\text { IIA: El tumor en la mama mide entre } 2 \text { y } 5 \mathrm{~cm} \text {. De uno a tres ganglios linfáticos axilares pueden estar invadidos. } \\
\text { IIB: El tumor en la mama mide más de } 5 \mathrm{~cm} \text { y las células anormales no han invadido los ganglios linfáticos axi- } \\
\text { lares. }\end{array}$ \\
\hline III & El tumor se ha propagado a la pared torácica o a la piel de la mama y ganglios axilares. \\
\hline IV & El cáncer se ha propagado a órganos distantes, como huesos, hígado, cerebro o pulmones. \\
\hline
\end{tabular}

\section{Tipos de tratamiento}

Respecto al tratamiento para el cáncer de mama, los artículos revisados indican que en cada mujer es diferente y depende del estadio de la enfermedad (Martínez et al., 2014)

Los tratamientos que se conocen son los siguientes: la radioterapia, la cual consiste en la aplicación de energía producida por un aparato de rayos X que ataca directamente las células malignas; la quimioterapia, que se basa en el uso de medicamentos que destruyen las células malignas; los tratamientos hormonales, que logran que las células cancerígenas no puedan reproducirse más, y la cirugía, que consiste en extirpar los tumores que se han producido en la mama. Los citados tratamientos pueden ser conservadores, radicales curativos, radicales paliativos y reconstructivos.

Para el control de este tipo de cáncer es de suma importancia sensibilizar a la población en general sobre la enfermedad en sí y de los mecanismos de control, así como también promover políticas y programas adecuados de prevención y diagnóstico temprano, pues el control integral del cáncer abarca la prevención, la detección precoz, el diagnóstico y tratamiento, la rehabilitación y los cuidados paliativos.

\section{Desarrollo de la ansiedad en mujeres con cáncer de mama}

La ansiedad surge ante situaciones desagradables, de resultado incierto o imprevisible que aumentan la alerta al prever un resultado potencialmente desfavorable a los intereses del sujeto. De aquí que se le considere como una reacción adaptativa cuya finalidad es la anticipación de una respuesta adecuada ante situaciones trascendentales. Sin embargo, aunque la ansiedad se suponga como una emoción natural de tipo adaptativo, también muestra un matiz patológico que puede afectar la vida personal, familiar, laboral o social del individuo cuando la frecuencia, intensidad o duración de la respuesta ansiosa resulta excesiva (Muñoz y Bernal, 2016).

La afectación del ritmo cardiaco se halla entre las expresiones fisiológicas de la ansiedad causadas por la afectación del sistema nervioso simpático y parasimpático, pues se torna más frecuente y al hacerlo provoca una mayor rapidez del torrente sanguíneo, además del aumento de la fuerza musculo-esquelética y demás síntomas físicos, como resequedad de la boca, sudoración en algunas partes del cuerpo, escalofríos, tensión muscular y dolor de pecho, entre muchos otros (Clark y Beck, 2012). Esos mismos autores plantean que el 
trastorno de ansiedad en los últimos años ha tenido una mayor incidencia, especialmente en el sexo femenino, y reconocen el interés que existe sobre su origen, curso y tratamiento, que han sido problemáticos a lo largo de los años. Lo anterior se conoce desde los estudios desarrollados por Sigmund Freud, en los que se habla de la neurosis, a la cual se asociaban algunos síntomas que correspondían a este desorden de ansiedad; sin embargo, con el paso del tiempo y la realización de diversas investigaciones, este trastorno ha logrado su propia clasificación dentro del Manual Diagnóstico y Estadístico de los Trastornos Mentales de la American Psychiatric Association (APA), en sus ediciones IV (1994) y V (2014).

Clark y Beck (2012) apuntan que el desarrollo del trastorno de ansiedad implica cinco criterios que afectan la vida de las personas: 1) elemento cognitivo disfuncional, en el que se estructura un pensamiento erróneo ante una situación que probablemente no suceda, pero que de alguna manera el sujeto se anticipa ante este probable evento; 2) deterioro del funcionamiento, que conlleva la alteración de la vida del sujeto, pues ante la ansiedad que se manifiesta ocurren alteraciones en su vida social que previenen la amenaza que supuestamente se puede generar; 3) persistencia, que ocasiona un aumento de la aprehensión subjetiva ante la amenaza potencial, dando un mayor valor a dicha situación aunque no suceda; 4) falsas alarmas, que ocurren en el desarrollo de la ansiedad, por las cuales el sujeto experimenta sensaciones de miedo o pánico debido a la sobrevaloración que hace de una amenaza, misma que puede ser inexistente pero que reviste gran importancia en virtud de que es concebida como real, y 5) hipersensibilidad al estímulo, la cual acarrea una reacción inmediata y quizá desproporcionada ante un evento que tal vez no se encuentra asociado directamente con la amenaza, pero que trae consigo distintas reacciones del individuo, tanto cognitivas, como emocionales y físicas.

De esta manera, el trastorno de ansiedad afecta directamente la vida de la persona que la padece al alterar de manera considerable su conducta y estilo de vida.

La ansiedad también aparece ante diversas enfermedades crónico-degenerativas, como el cáncer de mama, que afecta todas las áreas vitales de la mujer que lo padece; en efecto, muchos de los cambios que esta enfermedad genera alteran la vida psicológica, el desarrollo personal, la calidad de vida y la posición ante la enfermedad por el impacto emocional que atraviesan las pacientes; Martínez et al. (2014) señalan que tales reacciones pueden ser expresiones interpersonales o sociales de las emociones, entre las que se incluyen el miedo, la ira, el placer, la tristeza y otras, así como reacciones fisiológicas como el incremento de la tasa cardiaca, de la presión arterial sistólica y de la circulación sanguínea en músculos, o bien el decremento de la irrigación sanguínea de la piel.

Es importante mencionar que hay diferentes momentos en los que la ansiedad incide en las pacientes que sufren cáncer de mama: al recibir el diagnóstico, durante el tratamiento y principalmente durante las cirugías (Canicali, Nunes, Pires, Costa y Costa, 2012). En cuanto a la ansiedad que sufren las mujeres con dicha enfermedad, Muñoz y Bernal (2016) encontraron que la percepción de la cercanía de la muerte, las creencias sobre el cáncer, la anticipación del sufrimiento propio y de los allegados, la dificultad de expresar sus sentimientos, la sensación de pérdida de control, la crisis de las creencias y la necesidad de trascender, así como los problemas que aparecen en el entorno, carencia o exceso de cuidados y estimulación, el aburrimiento, los problemas de índole económica y asimismo las condiciones médicas -que son de suma importancia-, como la pérdida de vitalidad, las náuseas, la anorexia o los vómitos generados por los procedimientos a los que son sometidas desencadenan en las pacientes el temor a no recuperarse, y todo ello está relacionado con las alteraciones afectivas y de ansiedad. En el mismo sentido, se han hecho estudios en los que se han encontrado factores que incrementan los niveles de ansiedad durante el tratamiento, como el dolor severo (Muñoz y Bernal, 2016).

Se ha observado que durante los procedimientos a los que tienen que someterse -como la colocación del catéter puerto para la administración de la quimioterapia-, las pacientes manifiestan elevados niveles de ansiedad debido a la espera del procedimiento, la información insuficiente o equivocada, el miedo al dolor o el miedo a futuras complicaciones. Lugo, Becerra y Reynoso (2013) llevaron a cabo un estudio con mujeres con 
cáncer de mama en edades de 44 a 74 años que asistían a la Clínica de Terapia Intravenosa de la Unidad de Oncología del Hospital Juárez de México, en quienes midieron el grado de ansiedad, así como los signos y síntomas percibidos por ellas antes y después del procedimiento. Los autores concluyen que la mayoría de las pacientes se sintieron menos ansiosas al finalizar la colocación del catéter, y también encontraron que disminuye la percepción de ansiedad al llevarse a cabo intervenciones psicológicas antes, durante y después del procedimiento.

En pacientes mastectomizadas, el impacto que causa el procedimiento quirúrgico produce diferentes emociones, como frustración, tristeza e enojo, pero también, con mayor regularidad, trastornos de ansiedad y depresión, síntomas relacionados con el estrés al haber ya pensamientos intrusivos en la percepción del cáncer (Martínez et al., 2014).

Canicali et al. (2012) evaluaron la ansiedad "natural" en 84 mujeres mastectomizadas de 40 a 59 años mediante el Inventario de Ansiedad Indicio-Estado (IDATE) (versión brasileña del Inventario de Ansiedad Rasgo-Estado [IDARE] de Spielberger y Díaz-Guerrero [1975]), que identifica a las pacientes con mayor riesgo de desarrollar perturbaciones en el curso del tratamiento. Sus resultados indican que las pacientes mostraron un nivel medio en el indicio y el estado de ansiedad, resultados que se esperaban debido a que la población estaba vinculada a un programa de rehabilitación en el que se les proporcionaba atención interdisciplinaria.

Shin et al. (2016) estudiaron a 140 mujeres con cáncer de mama metastásico que recibían terapia endocrina o quimioterapia, en las cuales evaluaron la calidad de vida, la percepción de su pronóstico, la depresión y la ansiedad. Estos autores hallaron que las pacientes sometidas a quimioterapia mostraban tasas más elevadas de ansiedad y más síntomas depresivos en comparación con las pacientes tratadas con terapia endocrina. Dichas mujeres, que se consideraban a sí mismas como terminales, no manifestaron ansiedad, aunque sí depresión, y optaron por tratamientos que aliviaran principalmente su sintomatología.

Por otro lado, Yang et al. (2017) hicieron un estudio destinado a evaluar el riesgo de depresión, ansiedad y trastornos relacionados con el estrés en una cohorte nacional sueca de 40,849 mujeres con cáncer de mama invasivo y 4,402 mujeres con carcinoma mamario in situ. Considerando los posibles efectos dependientes del tiempo, encontraron que las mujeres con cáncer de mama invasivo mostraron mayores tasas de depresión, ansiedad y trastornos relacionados con el estrés. Los puntajes fueron mayores poco después del diagnóstico, pero continuaron aumentando hasta cinco años después de este. Las pacientes con carcinoma in situ exhibieron un aumento en la incidencia de trastornos relacionados con el estrés solo durante los primeros seis meses después del diagnóstico. Los autores destacan la importancia de la medición del tiempo ya que observaron perfiles de riesgo en las pacientes con cáncer de mama invasivo, lo que puede ser una guía significativa, oportuna y concreta en las intervenciones psicooncológicas.

Hayes et al. (2017) encontraron una interacción significativa entre el tiempo de espera ante la recomendación de una biopsia, la ansiedad y el estrés crónico, destacando que el tiempo se asoció con una mayor ansiedad en mujeres con bajos niveles de estrés; sin embargo, las mujeres que presentaron niveles altos de estrés experimentan mayor ansiedad, independientemente del tiempo de espera, concluyendo los autores que las mujeres pueden beneficiarse con tiempos de espera más cortos y de conocer estrategias adecuadas para el manejo de la ansiedad.

Del mismo modo, Guan et al. (2017) compararon los niveles de depresión y los de ansiedad en 221 pacientes con cáncer de mama. Sus resultados muestran que la sensación de ansiedad aparece con mayor frecuencia e intensidad que la de depresión.

Otra medición importante fue la realizada por Patel-Kerai, Harcourt, Rumsey, Naqvi y White (2017), quienes analizaron las diferencias entre 40 mujeres negras y 53 blancas del sur de Asia, sobrevivientes de cáncer de mama. Dichos autores encontraron diferencias significativas entre ambos grupos étnicos: las pacientes blancas reportaron mayores niveles de ansiedad y depresión, mayor deterioro de la calidad de vida y más creencias fatalistas relativas al cáncer; las mujeres negras, a su vez, obtuvieron niveles elevados de imagen corporal y más creencias religiosas en cuanto al 
control de su enfermedad. Los autores subrayan la importancia del apoyo cultural que se debe ofrecer a las pacientes.

\section{Calidad de vida en el cáncer de mama}

La Organización Mundial de la Salud (OMS) (2005) define la calidad de vida como un constructo multidimensional (salud física, estado psicológico, nivel de independencia, relaciones sociales, medio ambiente y preocupaciones espirituales) en el que se toman en cuenta las percepciones individuales en el contexto cultural y el sistema de valores. Diversos autores la han definido desde varias perspectivas: para algunos, la calidad de vida representa la capacidad del paciente para resolver los problemas que se relacionan con el hecho de estar gravemente enfermo; mientras que Sánchez, Ballesteros y Anzola (2010) la definen como el grado de satisfacción percibida con las circunstancias vitales actuales. Otras propuestas de multidimensionalidad plantean dominios tales como la capacidad para vivir una vida normal, la felicidad-satisfacción, el logro de metas personales, la capacidad para llevar una vida socialmente útil y las capacidades físicas y mentales. Sin embargo, todas estas definiciones son adaptaciones de la definición propuesta por la OMS.

Tal multiplicidad de definiciones, que inciden en la falta de claridad en el concepto, afectan a su vez su delimitación ya que algunos estudios han reportado que no existe correlación entre el estado de salud y la calidad de vida; así, es posible que los pacientes que sienten un compromiso importante con su estado de salud refieran una adecuada calidad de vida, o a la inversa: buenas condiciones de salud se relacionan con una mala calidad de vida; a esta situación particular se le denomina "paradoja de discapacidad", la cual se ha reportado en pacientes con cáncer al encontrarse que hay diferencias importantes entre la calificación de calidad de vida según el paciente, el médico y los familiares.

Recientes investigaciones versan sobre la asociación que hacen los pacientes oncológicos con la concepción de una buena o mala calidad de vida; así pues, la buena calidad de vida tiende a asociarse con una sensación de bienestar emocional, la percepción de una buena salud en el plano físico y psicológico, una convivencia familiar armónica que incluya un entorno cariñoso, la posibilidad de compartir con amigos, el contar con trabajo, tranquilidad económica, el disfrute de logros y la paz espiritual; como su contraparte, la mala calidad de vida se relaciona con tener una salud deficiente, malestar emocional, preocupaciones, limitaciones, convivencia familiar conflictiva, malos hábitos de alimentación, carencia de trabajo, deudas, dificultades en las relaciones sociales y sensación de angustia y desolación (Ríos y Cruzat, 2015).

Como ya se ha apuntado en los párrafos anteriores, hablar de calidad de vida es hacer referencia a un concepto multidimensional en cuanto que incorpora aspectos sociales, ambientales y creencias personales, de manera que se le define como "la evaluación que la persona realiza sobre su grado de bienestar o satisfacción en diversos dominios de la vida, considerando el impacto que en estos ha podido provocar una enfermedad física o mental y sus consecuencias" (Urzúa y Jarne, 2008, p. 151). Por ende, todo lo que la calidad de vida engloba se ve afectado de manera considerable cuando la persona se entera que padece cáncer, y más aún las mujeres con cáncer de mama.

Teniendo en cuenta lo anterior, se ha propuesto el término calidad de vida relacionada con la salud como una manera de describir aquellos aspectos de la experiencia subjetiva vinculados directa e indirectamente con la salud, la enfermedad, el tratamiento, la incapacidad y el deterioro.

En las mujeres con cáncer de mama se integran tres componentes en la percepción de su calidad de vida: el componente individual, el de interacción y el comunitario; el primero se refiere al estrés derivado de la enfermedad; el segundo alude al apoyo social de que dispone la paciente, y el último está relacionado con el sentido de pertenencia (Sánchez, Sierra y Martín, 2015). Por ello, al decir de Ríos y Cruzat (2015), la calidad de vida relacionada con la salud se refiere a la autovaloración que la paciente tiene de su habilidad para realizar actividades normales en su vida cotidiana y el impacto que la enfermedad y su tratamiento tienen en su estado de salud y en las distintas áreas de su vida. 
Para hacer un análisis de la calidad de vida en pacientes con cáncer de mama es importante tomar en cuenta la etapa del desarrollo vital en la que aquellas se encuentran, pues las necesidades y aspiraciones son diferentes en cada fase y de ello depende el tipo de afrontamiento que utilizan cuando se les informa el diagnóstico. Fernández, Llorens, García, Zabalza y Jorge (2009) indican que existen aspectos biológicos y psicológicos que afectan de manera considerable el desarrollo del cáncer durante la etapa de la adolescencia: en lo biológico aparecen trastornos del crecimiento e infertilidad, mientras que en el aspecto psicológico sobrevienen secuelas psicosociales que afectan el estilo de vida de las pacientes; de igual manera, la incidencia de depresión y el desarrollo del estrés postraumático son secuelas que suelen emerger durante y después de haber sufrido la enfermedad.

Otro cambio que de manera significativa surge en la vida de estas pacientes es en el ámbito escolar, pues cuando se diagnostica cáncer de mama de mujeres menores a 20 años de edad que se encuentran estudiando, en la mayoría de los casos hay efectos negativos en su desarrollo académico (Fernández et al., 2009). Otra de las áreas que sufre afectaciones es la relación familiar, toda vez que a menudo los familiares sobreprotegen a la paciente, limitando en consecuencia sus actividades.

Como indican Irarrazábal et al. (2016), las mujeres adultas también experimentan cambios significativos, como tener que dejar de trabajar debido a los diversos tratamientos, lo que hace que se deteriore su calidad de vida y aparezcan síntomas depresivos y ansiosos. Los estudios realizados por dichos autores han demostrado que en las pacientes sobrevivientes perviven algunas secuelas, lo que cambia en ocasiones su rutina; por ejemplo, el cansancio ocurre con mayor frecuencia, así como insomnio y otros problemas de ansiedad, los que de alguna medida alteran su vida cotidiana debido a los diversos tratamientos a los que son sometidas para resolverlos.

Un procedimiento que puede afectar la calidad de vida de las pacientes es la mastectomía, pues perder uno o ambos senos tiene implicaciones psicológicas difíciles de superar (Irarrazábal et al., 2016). Autores como Mols, Vingerhoets, Coebergh y Van de Poll-Franse (2005), Carlson et al. (2013) y Rodríguez y Font (2013) coinciden en señalar que los signos más frecuentes son el temor a la recidiva y la muerte, la sensación de pérdida de control, los problemas de adaptación a los cambios físicos, el malestar psicosocial, las dificultades en las relaciones interpersonales y sexuales (ya que la alteración de la simetría corporal es percibida por estas mujeres como una deformidad) y la preocupación respecto a su vida laboral.

Uno de los aspectos de la calidad de vida que se ve favorecido cuando sobrevienen las enfermedades crónico-degenerativas es el espiritual, y el cáncer de mama no es distinto; de hecho, numerosas pacientes suelen afrontar la enfermedad manifestando un mayor apego hacia su religión, lo que fortalece así este elemento primordial de la vida humana (García y Lucio 2016). Asimismo, la familia aparece como un apoyo importante para estos pacientes, al igual que la relación con los amigos, pues ello logra que la enfermedad se viva con pensamientos positivos, lo que propicia una mejor calidad de vida y fortalece el sistema inmunológico.

\section{Intervención psicológica}

El cáncer de mama por sí mismo, al igual que su tratamiento, generan un monto importante de ansiedad, lo que restringe de manera importante la vida social, familiar y personal de la mujer que lo padece, por lo que es necesario el trabajo psicológico. Según Rojas (2006), la intervención debe llevarse a cabo en cada etapa del proceso de enfermedad. El Cuadro 2 resume las diferentes etapas y los objetivos de la intervención en cada una de ellas.

Específicamente, la atención psicológica de las pacientes tiene como objetivos trabajar la imagen corporal (sobre todo en las pacientes mastectomizadas), el malestar y el sufrimiento emocional; disminuir el distrés psicosocial; prevenir la progresión hacia alguna condición psicopatológica, y contribuir al manejo de la ansiedad, la depresión, el estrés postraumático, el insomnio y los síntomas secundarios de la quimioterapia, o a la interrupción artificial de la menopausia. 
Cuadro 2. Intervención psicológica en el cáncer de mama.

\begin{tabular}{|c|c|c|}
\hline Etapa & Objetivo & Objetivo terapéutico \\
\hline Diagnóstico & $\begin{array}{l}\text { Facilitar la adherencia a los protocolos } \\
\text { médicos. }\end{array}$ & $\begin{array}{l}\text { Facilitar la percepción de control sobre la enfermedad y la } \\
\text { detección de las necesidades psicológicas y sociales. }\end{array}$ \\
\hline Tratamiento & $\begin{array}{l}\text { Controlar y manejar los efectos secunda- } \\
\text { rios psicológicos asociados al tratamiento } \\
\text { médico. }\end{array}$ & $\begin{array}{l}\text { Facilitar la adaptación a la enfermedad y a los tratamientos } \\
\text { médicos; fomentar estilos de afrontamiento activo y redes } \\
\text { de apoyo, y facilitar asimismo la adherencia terapéutica. }\end{array}$ \\
\hline $\begin{array}{l}\text { Intervalo libre } \\
\text { de enfermedad }\end{array}$ & $\begin{array}{l}\text { Ayudar a afrontar mejor la angustia en los } \\
\text { controles médicos, pues cada uno de ellos es } \\
\text { experimentado como una posible recaída. }\end{array}$ & $\begin{array}{l}\text { Alentar la expresión del miedo y de sus preocupaciones; } \\
\text { promover la buena comunicación médico-paciente, y facili- } \\
\text { tar el retorno de la paciente a su vida cotidiana. }\end{array}$ \\
\hline Supervivencia & $\begin{array}{l}\text { Tratar las dificultades relacionadas directa o } \\
\text { indirectamente con la enfermedad, mismas } \\
\text { que puedan seguir causando alteraciones } \\
\text { emocionales. }\end{array}$ & $\begin{array}{l}\text { Facilitar la adaptación a las secuelas físicas, psicológicas } \\
\text { y sociales, así como la reincorporación a las actividades } \\
\text { cotidianas de la paciente y a su vida sexual. }\end{array}$ \\
\hline Recurrencia & $\begin{array}{l}\text { Intervenir en el impacto psicológico, que } \\
\text { puede ser más intenso que en el diagnóstico } \\
\text { inicial. }\end{array}$ & $\begin{array}{l}\text { Abatir síntomas como la ansiedad, la angustia extrema y la } \\
\text { depresión; facilitar la adaptación al nuevo estado de la en- } \\
\text { fermedad; reforzar la relación médico-paciente, y detectar } \\
\text { las necesidades emocionales de la familia. }\end{array}$ \\
\hline Final de la vida & $\begin{array}{l}\text { Intervención en las reacciones emociona- } \\
\text { les que aparecen con gran intensidad en la } \\
\text { paciente y en su familia. }\end{array}$ & $\begin{array}{l}\text { Colaborar en el control de los síntomas físicos; detectar y } \\
\text { atender dificultades psicológicas y sociales que surjan en } \\
\text { la paciente y en su familia; acompañar a la paciente en el } \\
\text { proceso de morir y facilitar el duelo en los familiares. }\end{array}$ \\
\hline
\end{tabular}

Fuente: Rojas (2006).

En los últimos años se han propuesto diferentes formas de apoyo psicológico, al igual que varios tipos de terapias para las pacientes con cáncer de mama. Ascencio, Rangel y Allende (2013), Vázquez et al. (2014) y Alga y García (2016) coinciden en que las estrategias de intervención psicológica en el cáncer de mama más utilizadas son las siguientes:

1) Terapias cognitivo-conductuales (entrenamiento en estrategias para el manejo de la ansiedad, exposición gradual a los estímulos temidos, exposición interoceptiva, técnicas de distracción, entrenamiento en resolución de problemas y habilidades de comunicación, reestructuración cognitiva, ventilación emocional, reducción del estrés basado en el mindfulness [Andersen et al., 2013] y escucha activa). Estas son de utilidad para eliminar pensamientos depresivos, autocondenatorios, de culpa, desesperanza o de autocompasión mediante la modificación de la forma de razonamiento que emplea la paciente por medio de la autoobservación, el automonitoreo y la recapacitación (Suárez y Almanza, 2017).

2) Intervención psicoeducativas. Algunos autores como Vázquez et al. (2014) consideran que la misma, en combinación con estrategias como la rehabilitación física y la modificación de la dieta, es de las intervenciones más efectivas para la reducción de la sintomatología psicológica del cáncer de mama.

3) Psicoterapia breve. Útil para abordar de manera directa los problemas de separación, cambio de estilo de vida y sentimiento de pérdida; su objetivo es disminuir el malestar psicosocial y contribuir a que las pacientes recobren la funcionalidad, retomen el control de su vida al incorporar, con el menor sufrimiento posible, las distintas intervenciones médicas que su enfermedad exige (Moncayo y Breitbart, 2013; Suárez y Almanza, 2017).

4) Psicoterapia de grupo. Útil a todo lo largo del proceso de atención oncológica, pero está limitada a los pacientes cuyo estado clínico les permite físicamente acudir o ser llevados al espacio grupal. Existen estudios que indican que la terapia de grupo para las pacientes facilita el cambio de actitudes, conductas y emociones por medio de la reflexión conjunta, el intercambio de experiencias, el autoconocimiento y el apoyo mutuo. Por los resultados obtenidos, la intervención de grupo se recomienda para mejorar la calidad de vida de las mujeres supervivientes del cáncer de mama (Rodríguez y Font, 2013).

5. Terapias alternativas y complementarias. De ellas, las que más destacan son aquellas que 
involucran cuerpo y mente (técnicas de relajación, visualización, yoga). En algunos países europeos se emplean en $35.9 \%$ de los casos con el propósito de mejorar el bienestar físico o psicológico y la capacidad del cuerpo para combatir el cáncer. Se aplican en situaciones de ansiedad (36\%), para el miedo (29.4\%) y para el dolor (25\%) (León et al., 2013).

6) Intervención psicológica al final de la vida. Es complicada pero absolutamente necesaria, pues en ella convergen de manera definitiva todos los factores (complicaciones asociadas o paralelas al cáncer y los aspectos psicosociales en toda su complejidad) que a lo largo del proceso de la atención de la enfermedad han tenido lugar. Su objetivo es ayudar a lograr aceptación y tranquilidad para poder cerrar los aspectos que generan intranquilidad a la paciente en la etapa final de su vida (Rojas, 2006).

Todos estos tipos de intervención psicológica tienen como objetivo incidir en la mejora de la sintomatología emocional, favorecer la adaptación a esta condición de salud y mejorar la calidad de vida de la mujer con diagnóstico de cáncer de mama.

\section{DISCUSIÓN}

Como resultado de la revisión llevada a cabo en este trabajo, se puede señalar que el cáncer de mama es una de las neoplasias que ocurren con mayor frecuencia en la mujer en todo el orbe. A pesar de los actuales avances médicos para tratar este padecimiento, sigue siendo una patología que tiene un pronóstico incierto, dolorosa en los ámbitos personal y de pareja, compleja para las familias de las mujeres que la sufren, y en ocasiones muy costosa.

La mayoría de los estudios referentes a dicha patología señalan que las mujeres diagnosticadas con cáncer de mama experimentan altos niveles de ansiedad que se acompañan de un proceso psicológico adaptativo a la enfermedad denominado "duelo oncológico", que consiste en una serie de etapas que no necesariamente se presentan en forma secuenciada y que comienzan durante los meses siguientes al diagnóstico, duelo que tiene como objetivo la aceptación y la adaptación a esta condición de salud. En este mismo sentido, la literatura revisada muestra que las manifestaciones de ansiedad están relacionadas con tres aspectos: los efectos que pueden tener los tratamientos, los cuales se asocian a la pérdida de control de la propia vida, al impacto en la calidad de esta y a la asociación que se hace de la enfermedad con la posibilidad de muerte. En relación a ello, se puede decir que el grado de malestar que puede experimentar la mujer afectada por el cáncer mamario reúne aspectos biológicos, sociales, ambientales y espirituales.

Asimismo, en los estudios revisados se afirma que la ansiedad se encuentra presente durante el diagnóstico, el tratamiento, los procedimientos quirúrgicos $\mathrm{y}$, cuando ocurre, en la recurrencia de la enfermedad.

Por otro lado, los trabajos referentes a las manifestaciones de ansiedad ante los tratamientos señalan que la mastectomía es un proceso al que se le da particular importancia porque la mama es un órgano que tiene una representación psicológica, sexual, afectiva y cultural especial y compleja; por tanto, el diagnóstico de cáncer en este órgano, su tratamiento y las secuelas que resultan son una de las principales fuentes generadoras de angustia en las pacientes, misma que provoca cambios en lo biológico y lo psicológico y altera así su calidad de vida. En lo que respecta a este concepto, es posible subrayar que, ante la enfermedad, la percepción que tiene la paciente de su vida se modifica drásticamente, lo que afecta desfavorablemente su bienestar emocional en cuanto que asume su condición médica como el principal núcleo de tensión y malestar, y progresivamente generaliza esta percepción negativa hacia otras dimensiones, causando así graves afectaciones a su calidad de vida.

La aparición de la sintomatología depresiva y ansiosa en las pacientes diagnosticadas con cáncer de mama es una respuesta hasta cierto punto normal, pero es necesario brindar la atención psicológica adecuada para prevenir que dicha sintomatología emocional rebase sus recursos de afrontamiento y genere un deterioro mayor en su calidad de vida y en la adaptación a la enfermedad. Por ello, el objetivo principal de la psicoterapia es ayudar a las mujeres a sobrellevar los cambios físicos y emocionales y en su estilo de vida relacionados con el 
cáncer, así como los tratamientos médicos, mismos que pueden resultar dolorosos y traumáticos.

Finalmente, es importante señalar que el manejo efectivo de la ansiedad es de suma importancia para las mujeres que sufren esta enfermedad, pues les ayuda a lidiar de una manera más adapta- tiva con el dolor, el miedo y demás emociones que afectan de manera negativa su calidad de vida.

Por lo anterior, se sugiere que futuras investigaciones profundicen el estudio de la ansiedad en pacientes diagnosticados con cáncer.

\section{REFERENCIAS}

Alga M., J. y García P., B. (2016). Abordaje de la ansiedad en pacientes diagnosticados de cáncer. Psicooncología, 13(2-3), 227248. doi: 10.5209/PSIC.54434.

American Psychiatric Association (1994). Diagnostic and Statistical Manual of Mental Disorders (IV ed.). Washington, D.C.: APA. American Psychiatric Association (2014). Diagnostic and Statistical Manual of Mental Disorders (V ed.). Washington, D.C.: APA.

Andersen, S.R., Würtzen, H.S.J., Christensen, J., Andersen, K.K., Flyger, H. y Dalton, S.O. (2013). Effect of mindfulness-based stress reduction on sleep quality: Results of a randomized trial among Danish breast cancer patients. Acta Oncologica, 52(2), 336-344. doi: 10.3109/0284186X.2012.745948.

Ascencio H., L., Rangel D., N.E. y Allende P., S. ( 2013). Proceso de atención psicológica en el Programa de Atención y Cuidados en Oncología del Instituto Nacional de Cancerología. Psicooncología, 10(273), 393-406. doi: 10.5209/rev_PSIC.2013.v10. n2-3.43457.

Canicali, P., Nunes, G., Pires, O., Costa, L. y Costa, A. (2012). Ansiedad en mujeres con cáncer de mama. Enfermería Global. 28(10), 52-62.

Carlson, L.E., Doll, R., Stephen, J., Faris, P., Tamagawa, R., Drysdale, E., et al. (2013). Randomized controlled trial of mindfulness-based cancer recovery versus supportive expressive group therapy for distressed survivors of breast cancer (MINDSET). Journal of Clinical Oncology, 31, 3119-3126. doi: 10.1200/ JCO.2012.47.5210.

Clark, D. y Beck, A. (2012). Terapia cognitiva para trastornos de ansiedad. Bilbao: Desclée de Brouwer.

Fernández, B., Llorens, Á., García, R., Zabalza, A. y Jorge, V. (2009). Calidad de vida y estado de salud en niños y en adolescentes supervivientes de cáncer. Psicooncología, 6(2-3), 459-468.

García G., A.A. y Lucio G.M., E. (2016). Estilo de afrontamiento y calidad de vida en adolescentes con cáncer. Gaceta Mexicana de Oncología, 15(1), 3-10. Doi: 10.1016/j.gamo.2016.02.004 1665-9201.

Gonzalez, E., Garcia, P., Aguilar, M., Padilla, C. y Alvarez, J. (2014). Breastfeeding and the prevention of breast cancer: a retrospective review of clinical histories. Clinical Nursing, 23, 2397-2403. doi: 10.1111/jocn.12368.

Guan, N.G., Mohamed, S., Kaur, K., Hatim, S.A., Zuraida, Z.N. y Aishah, T.N. (2017). Perceived distress and its association with depression and anxiety in breast cancer patients. Plos One, 12(3), e0172975. doi: 10.1371/journal.pone.0172975.

Hayes, B.M., Shelby, S.R., Wren, A.A., Miller, S.L., Yoon, C.S., Baker, A.J., et al. (2017). Anxiety prior to breast biopsy: Relationships with length of time from breast biopsy recommendation to biopsy procedure and psychosocial factors. Journal of Health Psychology, 22(5), 561-571. doi: 101177/1359105315607828.

Irarrázabal, M., Kleinman, P., Silvar, F., Fritis, M., Barriga, C. y Waintrub, H. (2016). Calidad de vida en pacientes chilenas sobrevivientes de cáncer de mama. Revista Médica de Chile, 144(12), 1567-1576. doi: doi.org/10.4067/S003498872016001200008.

Kern, D. y Moro, L. (2012). Factores psicosociales relacionados con el autocuidado en la prevención, tratamiento y postratamiento del cáncer de mama. Psiconcología, 9(2-3), 453-465. doi: 10.5209/rev_PSIC.2013.v9.n2-3.40916.

León, C., Mirapeix, R., Blasco, T., Jovell, E., Arcusa, À., Martín, A. y Seguí, M.À. (2013). Mindfulness para la reducción del malestar emocional en pacientes oncológicos. Estudio comparativo con una intervención psicoeducativa estándar. Psicooncología, 10(2-3), 263. doi: 10.5209/rev_PSIC.2013.v10.n2-3.43448.

Lugo, G., Becerra, G. y Reynoso, E. (2013). Intervención psicológica para manejo de ansiedad ante la colocación de catéter puerto en mujeres con cáncer de mama. Psicooncología, 10(1), 177-184. doi: 10.5209/rev_PSIC.2013.v10.41956

Lukong, K. (2017). Understanding breast cancer - The long and winding road. BBA Clinical, 7, 64-77. doi: 10.1016/j.bbacli.2017.01.001.

Martínez B., A.E., Lozano A., A., Rodríguez V., A.L., Galindo V., O. y Alvarado A., S. (2014). Impacto psicológico del cáncer de mama y la mastectomía. Gaceta Mexicana de Oncología, 13(1), 53-58.

Mols, F., Vingerhoets, A.J., Coebergh, J.W. y Van de Poll-Franse, L.V. (2005). Quality of life among long-term breast cancer survivors: A systematic review. European Journal of Cancer, 41, 2613-2619. doi: 10.1016/j.ejca.2005.05.017. 
Moncayo F., L. y Breitbart, W. (2013). Psicoterapia centrada en el sentido: "Vivir con sentido". Estudio piloto. Psicooncología, 10(2/3), 233-245. doi: 10.5209/rev_PSIC.2013.v10.n2-3.43446.

Muñoz, A. y Bernal, G. (2016). Abordaje de la ansiedad en pacientes diagnosticados de cáncer. Psicooncología, 13(2-3), 227-248. doi: $10.5209 /$ PSIC.54434.

Organización Mundial de la Salud (2005). Calidad de vida. Ginebra: OMS.

Patel-Kerai, G., Harcourt, D., Rumsey, N., Naqvi, H. y White, P. (2017). The psychosocial experiences of breast cancer amongst Blacks, South Asian and White survivors: do differences exist between ethnic groups? Psicooncología, 26(4), $515-522$.

Ríos, M. y Cruzat, C. (2015). Percepción y significados asociados a la calidad de vida en pacientes hemato-oncológicos. Revista Chilena de Neuro-Psiquiatría, 53(4), 261-268. doi: 10.4067/S0717-92272015000400006.

Rodríguez, E. y Font, A. (2013). Eficacia de la terapia de grupo en cáncer de mama: evolución de las emociones desadaptativas. Psicooncología, 10(2-3), 275-287. doi: 10.5209/rev_PSIC.2013.v10.n2-3.43449.

Rojas M., G. (2006). Estrategias de intervención psicológica en pacientes con cáncer de mama. Revista Médica Clínica Las Condes, 17(4), 194-197.

Sánchez P., R., Ballesteros M., P. y Anzola J., D. (2010). Concepto de calidad de vida en mujeres con cáncer. Revista de Salud Pública, 12(6), 889-902.

Sánchez, R., Sierra, F. y Martín, E. (2015). ¿Qué es calidad de vida para un paciente con cáncer? Avances en Psicología Latinoamericana, 33(3), 321-385. doi: dx.doi.org/10.12804/ap133.03.2015.01.

Shin, J., El-Jawahri, A., Parkes, A., Schleicher, M., Knight, P. y Teme, S. (2016). Quality of life, mood, and prognostic understanding in patients with metastatic breast cancer. Journal of Palliative Medicine, 19(8), 863-869. doi: 10.1089/jpm.2016.0027.

Spielberger, C.D. y Díaz-Guerrero, R. (1975). IDARE: Inventario de Ansiedad Rasgo-Estado. México: El Manual Moderno.

Suárez I., R. y Almanza M., J.D. (2017). Abordaje psicoterapéutico en pacientes con cáncer de mama. Neurología, Neurocirugía y Psiquiatría, 44(4), 133-141.

Torres, G., Ortega, C., Ángeles, A., Villalobos, A.L., Salmerón, J., Lazcano, E. y Hernández, M. (2013). Patrones de utilización de programas de prevención y diagnóstico temprano de cáncer en la mujer. Salud Pública de México, 55, S241-S248.

Urzúa M., A. y Jarne E., A. (2008). Calidad de vida y estilos de afrontamiento en personas con patologías crónicas. Revista Interamericana de Psicología/Interamerican Journal of Psychology, 42(1), 151-160.

Vázquez O., G., Castillo E., R., Benjet, C., García A., M., Ponce J., L. y Aguilar S., A. (2014). Efectos de intervenciones psicológicas en sobrevivientes de cáncer: una revisión. Psicooncología, 11(2-3), 233-241. doi: 10.5209/rev_PSIC.2014.v11. $\mathrm{n} 2-3.47385$.

Vich, P., Brusint, B., Álvarez H., C., Cuadrado R., C., Díaz G., N. y Redondo, E. (2014). Actualización del cáncer de mama en atención primaria (I/V). Semergen, Medicina de Familia, 40(6), 326-333. doi: 10.1016/j.semerg.2014.02.012.

Yang, H., Brand, S., Fang, F., Chiesa, F., Johansson, L., Hall, P. y Czene, K. (2017). Time-dependent risk of depression, anxiety, and stress-related disorders in patients with invasive and in situ breast cancer. International Journal of Cancer, 140, 841852. doi: 10.1002/ijc.30514. 